\title{
Effect of Dilute Acid - Alkaline Pretreatment on Rice Husk Composition and Hydrodynamic Modeling with CFD
}

\author{
Novia $^{1 *}$, Vishnu K Pareek ${ }^{2}$, Hermansyah ${ }^{3}$, Asyeni Miftahul Jannah ${ }^{1}$ \\ ${ }^{1}$ Departement of Chemical Engineering, Faculty of Engineering, Universitas Sriwijaya, Indonesia \\ ${ }^{2}$ Western Australian School of Mines: Minerals, Energy and Chemical Engineering, Curtin University, Australia \\ ${ }^{3}$ Departement of Chemical Science, Mathematics and Science Faculty, Universitas Sriwijaya, Indonesia \\ *Corresponding author: novia@ft.unsri.ac.id
}

\begin{abstract}
The high cellulosic content of rice husk can be utilized as a feedstock for pulp and biofuel. Pretreatment is necessary to break the bonds in the complex lignocellulose matrices addressing the cellulose access. This work aims to utilize the rice husk using dilute acid and alkaline pretreatment experimentally and CFD modeling. The study consists of three series of research. The first stage was the dilute acid pretreatment with sulfuric acid concentration of $1 \%$ to $5 \%(\mathrm{v} / \mathrm{v})$ at $85^{\circ} \mathrm{C}$ for 60 minutes, and alkaline pretreatment with $\mathrm{NaOH}$ concentration of $1 \%$ to $5 \%(\mathrm{w} / \mathrm{v})$ at $850 \mathrm{C}$ for 30 minutes separately. The second stage used the combination of both pretreatment. Moreover the last stage of research was hydrodynamic modeling of pretreatment process by CFD (ANSYS FLUENT 16). The experimental results showed that the lowest lignin content after acid pretreatment was about $10.74 \%$. Alkaline pretreatment produced the lowest lignin content of $4.35 \%$. The highest cellulose content was $66.75 \%$ for acid-alkaline pretreatment. The lowest content of lignin was about $6.09 \%$ for acid-alkaline pretreatment. The lowest performance of alkaline pretreatment on HWS (hot water solubility) of about $7.34 \%$ can be enhanced to $9.71 \%$ by using a combination alkaline-acid. The combined pretreatments result hemicellulose of about $9.59 \%$ (alkaline-acid) and $9.27 \%$ (acid-alkaline). Modeling results showed that the mixing area had the minimum pressure of about $-6250 \mathrm{~Pa}$ which is vortex leading minimum efficiency of mixing. The rice husk flowed upward to the upper level and mixed with reagent in the perfect mixing.
\end{abstract}

Keywords

Alkaline Pretreatment, CFD Modeling, Dilute Acid pretreatment, Lignin, Rice Husk

Received: 17 October 2018, Accepted: 12 January 2019

https://doi.org/10.26554/sti.2019.4.1.18-23

\section{INTRODUCTION}

Programs on energy utilization have been sustained by finding other kinds of renewable energy. Several studies relating to the conversion of lignocellulose biomass as a raw material to produce bioethanol have been implemented (Chen et al., 2011; Hermansyah et al., 2015; Karimi et al., 2006; Novia et al., 2017; Sudiyani et al., 2017; Xavier et al., 2010).

The conversion of bioethanol is still challenging. The lignin component in lignocellulose biomass must be eliminated in order to enable the digestibility of enzymatic hydrolysis. Pretreatment is the most important step to reduce lignin and hemicellulose, to decrease the crystallinity of cellulose, and to improve the materials porosity (Karimi et al., 2006). It is needed to change the structure of cellulosic biomass, making the cellulose more porous to enzymes which can alter the polymers of carbohydrate into fermentable sugars (Mosier, 2005). After pretreatment, cellulose and hemicellulose are hydrolyzed to monomeric sugars. The pretreatment needs higher cost, so the best pretreatment can reduce the amount of chemicals for hydrolyzed. Pretreatment also enhance the glucose yield. Unpretreated samples gives lower than $20 \%$, using the pretreatment main process the glucose yield to about $90 \%$ (Hamelinck et al., 2005).

Methods for the pretreatment of lignocellulose biomass to increase the hydrolysis have been studied: steam explosion and alkaline peroxide (Cara, 2006; Fang et al., 2010; Ohgren et al., 2007); alkaline pretreatment (Harun et al., 2011; McIntosh and Vancov, 2010); and hydrothermal pretreatment (Kaparaju and Felby, 2010; Kumar et al., 2011; Thomsen et al., 2008). These technologies use extreme operating conditions of reaction with a greatly capital cost, highly processing fees and large investment risks (Li et al., 2009). Several pretreatment methods create strong acidic, poisonous materials in the preserved lignocellulose and inhibit mixtures for hydrolysis and following fermentation of ethanol. In addition, alkaline pretreatment was demonstrated to be more suitable on agricultural residues 
than on materials of wood (Taherzadeh et al., 2008). It also selectively reduces the lignin portion. Pretreatment of alkali apply at lower temperatures and pressures compared to other pretreatment methods (Mosier, 2005). The lime pretreatment process uses slurring the lime with water, showering it onto the biomass material and keeping the material for a particular duration, generally from days to weeks (Mosier, 2005). Ammonia has been usually used in alkali pretreatments; its use produces many environmental problems due to the recovering processes that must also be carried out along with this pretreatment. Reducing hemicellulose and lignin content could highly increase enzyme digestibility by enhancing the enzyme accessibility to cellulose. Pretreatment with dilute sulfuric acid followed by aqueous ammonia would then be required for the effective hydrolysis and lignin separation. With the benefits of dilute acid and alkaline method, this work investigated the combination of dilute acid-alkaline pretreatment to eliminate the lignin component in rice husk. Pretreatment is a high cost process to allow lignocellulose biomass to be utilized to become an alternative fuels. However it has the possibility to increase the effectiveness and decreasing of the fee through more study and advance.

Hydrodynamic simulation of the alkaline-dilute acid pretreatment was performed using the computational fluid dynamics (CFD) modeling (ANSYS FLUENT 16). The fundamental processes that occur in the pretreatment process have been investigated. Numerous researches (Novia et al., 2007, 2017; Cundari, 2016) are being applied to advance the computational methods appropriate for pairing the many crucial characteristics of chemistry and physics in a procedure that is effective enough for explaining the pretreatment problems. CFD modeling can offer a huge scope of information for the design of pretreatment reactor. The use of CFD modeling can therefore decrease the cost of time-consuming experimental research.

\section{EXPERIMENTAL SECTION}

\subsection{Materials}

The rice husk samples were obtained from nearby (South Sumatera). They were stored in plastic bags at $50 \mathrm{C}$, after determination of moisture content. The rice husks were collected from the location of Inderalaya, South Sumatera province, Indonesia. Then they were air-dried and kept at room temperature. They were crushed and put through a sieve tray (less than 0.8 $\mathrm{mm})$.

\subsection{Methods}

2.2.1 Experimental Method of Dilute Acid Pretreatment Lignocellulose rice husks were pretreated by the following procedure. Each dry rice husk, of about $30 \mathrm{~g}$, was fed into a stirred reactor of $500 \mathrm{~mL}$ and blended with $150 \mathrm{ml}$ of sulfuric acid $\left(\mathrm{H}_{2} \mathrm{SO}_{4}\right)$ with various concentration of $1 \%$ to $5 \%(\mathrm{v} / \mathrm{v})$. The mixture was kept warm in a rotary air bath at a speed of 500 $\mathrm{rpm}$ and temperature of $85^{\circ} \mathrm{C}$ for $30 \mathrm{~min}$. The mixture was inserted in an autoclave at a temperature of $121^{\circ} \mathrm{C}$ for $30 \mathrm{~min}$. The mixture was then put into $1 \mathrm{~L}$ of pre-cold acetone and blended carefully. The slurry was separated using centrifuge at a speed of $8000 \mathrm{rpm}$. After $10 \mathrm{~min}$ of time, the filtrate was gathered. The residue remains was rinsed in $1 \mathrm{~L}$ of water and separated. The component of lignin before and after dilute-acid pretreatment was analyzed by a standard method of Chesson (Datta 1981). Scanning electron microscope (SEM) micrographs of cross section rice husk before and after pretreatment were taken using SEM-EDX microscope Phenom Pro X.

\subsubsection{Experimental Method of Dilute Acid-Alkaline Pre- treatment}

Dilute-acid pretreated samples were followed by alkaline pretreatment to improve the removal percentage of lignin content in the samples

\subsubsection{Experimental Method of Alkaline-Dilute Acid Pre- treatment}

Alkaline pretreated samples were followed by dilute-acid pretreatment to reduce percentage of lignin content in the samples

\subsubsection{Modeling of Dilute Acid-Alkaline Pretreatment by Us- ing CFD}

The CFD simulations were carried out by the Euler-Granular multiphase model available in the commercial CFD software (ANSYS FLUENT 16.0 Documentation 2015). Simplification assumption is each phase coincides at every grid in domain in the form of interpenetrating continua.

The mass conservation of phase $i(i=$ fluid, solid $)$ :

$$
\frac{\partial}{\partial t}\left(\rho_{i} \varepsilon_{i}\right)+\nabla \bullet\left(\rho_{i} \varepsilon_{i} U_{i}\right)=r_{i}
$$

where, $\varepsilon_{f}+\varepsilon_{s}=1$

The conservation of momentum of phase $\mathrm{i}(\mathrm{i}=$ fluid, $\mathrm{k}=$ solid, $\mathrm{k} \neq \mathrm{i}$ ):

$\frac{\partial}{\partial t}\left(\rho_{i} \varepsilon_{i} U_{i}\right)+\nabla \bullet\left(\rho_{i} \varepsilon_{i} U_{i} U_{i}\right)=-\varepsilon_{i} \nabla P+\nabla \bullet \tau_{i}+\rho_{i} \varepsilon_{i} g-\beta\left(U_{i}-U_{k}\right)$

The conservation of energy for phase i gives:

$$
\frac{\partial}{\partial t}\left(\varepsilon_{i} \rho_{i} H_{i}\right)+\nabla \bullet\left(\varepsilon_{i} \rho_{i} U_{i} H_{i}\right)=-\varepsilon_{i} \frac{\partial P_{i}}{\partial t}+\tau_{i}: \nabla U_{i}-\nabla q_{i}+S_{i}
$$

with,

$$
H_{i}=\sum_{i} \varepsilon_{i} \int_{T_{r e f}}^{T} C p_{i} d T
$$

For the drag force formulation, we use the Syamlal-O'Brian model (ANSYS FLUENT 16.0 Documentation 2015) :

$$
\beta=\frac{3}{4} C_{D} \frac{\varepsilon_{s} \varepsilon_{g}}{v^{2}{ }_{r, s}} \frac{\rho_{g}}{d_{s}}\left(\frac{R e_{s}}{v_{r, s}}\right)\left|U_{s}-U_{g}\right|
$$


The drag coefficient, $\mathrm{C}_{\mathrm{D}}$ is given by:

$$
\begin{aligned}
& C_{D}=\left(0.63+\frac{4.8}{\sqrt{\frac{R e_{s}}{v_{r, s}}}}\right)^{2} \\
& R e_{s}=\frac{\rho_{g} d_{s}\left|U_{s}-U_{g}\right|}{\mu g}
\end{aligned}
$$

The terminal velocity correlation for the solid phase:

$v_{r, s}=0.5 A-0.03 R e_{s}+0.5 \sqrt{\left(0.06 R e_{s}\right)^{2}+0.12 R e_{s}(2 B-A)+A^{2}}$

where,

$$
\begin{aligned}
& A=\varepsilon_{g}^{4.14} \\
& B=0.8 \varepsilon_{g}^{1.28} \text { for } \varepsilon_{g} \leq 0.85 \\
& B=0.8 \varepsilon_{g}^{2.65} \text { for } \varepsilon_{g}>0.85
\end{aligned}
$$

Solid Pressure. The solids phase pressure (Ps) contains a kinetic term and the particle collisions term.

$$
P_{s}=\left(1+2\left(1+e_{s}\right) e_{s} g_{o}\right) \varepsilon_{s} \rho_{s} \Theta_{s}=\rho_{s} \varepsilon_{s} \Theta_{s}+2 g_{o} \rho_{s} \varepsilon_{s}^{2} \Theta_{s}\left(1+e_{s}\right)
$$

were $\Theta \mathrm{s}$ is the granular temperature, es is the coefficient of restitution for particle collisions. The radial distribution function, go, is defined by:

$$
g_{o}=\left[1-\left(\frac{\varepsilon_{s}}{\varepsilon_{s, \text { max }}}\right)^{\frac{1}{3}}\right]^{-1}
$$

Solids Shear Stress. The solids stress tensor contains bulk and shear viscosities. The solid phase bulk viscosity are expressed as:

$$
\mu_{b}=\frac{4}{3} \varepsilon_{s} \rho_{s} d_{s} g_{o}\left(1+e_{s}\right)\left(\frac{\Theta_{s}}{\pi}\right)^{\frac{1}{2}}
$$

The solids phase shear viscosity is given by:

$$
\mu_{b}=\frac{2 \mu_{s, d i l}}{(1+e) g_{o}}\left[1+\frac{4}{5}\left(1+e_{s}\right) g_{o} \varepsilon_{s}\right]^{2}+\frac{4}{5} \varepsilon_{s} \rho_{s} d_{s} g_{o}\left(1+e_{s}\right)\left(\frac{\Theta_{s}}{\pi}\right)^{\frac{1}{2}}
$$

The solid phase dilute viscosity is:

$$
\mu_{s, d i l}=\frac{5}{16} \rho_{s} \varepsilon_{s} l_{s} \sqrt{2 \pi \Theta_{s}}
$$

where is,

$$
l_{s}=\frac{\sqrt{2}}{12} \frac{d_{s}}{\varepsilon_{s}}
$$

The granular temperature, $\Theta_{s}$, is calculated by solving the turbulent kinetic energy equation for solid phase:

$$
\frac{3}{2} \frac{\partial}{\partial t}\left(\rho_{s} \varepsilon_{s} \Theta_{s}\right)+\nabla\left(\rho_{s} \varepsilon_{s} U_{s} \Theta_{s}\right)=T_{s}: \nabla U_{s}+\nabla\left(k_{\Theta_{s}} \nabla \Theta_{s}\right)-\gamma_{s}
$$

The diffusion coefficient for granular energy $\mathrm{k} \Theta_{s}$ is given by:

$$
k_{\Theta_{s}}=\frac{2 k_{\Theta_{s, d i l}}}{\left(1+e_{s}\right) g_{o}}\left(1+\frac{6}{5}\left(1+e_{s}\right) g_{o} \varepsilon_{s}\right)^{2}+2 \varepsilon_{s}^{2} \rho_{s} d_{s} g_{o}\left(1+e_{s}\right)\left(\frac{\Theta_{s}}{\pi}\right)^{\frac{1}{2}}
$$

where:

$$
k_{\Theta_{s, d i l}}=\frac{75}{64} \rho_{s} \varepsilon_{s} l_{s} \sqrt{2 \pi \Theta_{s}}
$$

The collisional energy dissipation, $\gamma \mathrm{s}$, is calculated by:

$$
\gamma_{s}=3\left(1-e_{s}^{2}\right) \varepsilon_{s}^{2} \rho_{s} g_{0} \Theta_{s}\left[\frac{4}{d_{s}}\left(\frac{\Theta_{s}}{\pi}\right)^{\frac{1}{2}}-\nabla U_{s}\right]
$$

Generally, the mixer is below turbulent flow conditions. Therefore, it is crucial to apply the suitable turbulence model to illustrate the effect of turbulent fluctuations of velocities and scalar variables for the basic conservation equations. A k- $\epsilon$ model was used to show the turbulent motions in both phases. In the $\mathrm{k}-\epsilon$ model, the turbulent viscosity is given by:

$$
\mu_{t, i}(t)=\rho_{i} \varepsilon_{i} C_{\mu} \frac{k_{i}^{2}}{\epsilon_{i}}
$$

The turbulence kinetic energy, $\mathrm{k}$, and its rate of dissipation, $\epsilon$, can be determined from the transport equations:

$$
\frac{\partial}{\partial t}\left(\rho_{i} \varepsilon_{i} k_{i}\right)+\nabla\left(\rho_{i} \varepsilon_{i} k_{i} U_{i}\right)=\nabla\left(\varepsilon_{i} \frac{\mu_{t}}{\sigma_{k}} \nabla k_{i}\right)+\left(\varepsilon_{i} G_{k}-\varepsilon_{i} \rho_{i} \epsilon_{i}\right)
$$

$$
\frac{\partial}{\partial t}\left(\rho_{i} \varepsilon_{i} \epsilon_{i}\right)+\nabla\left(\rho_{i} \varepsilon_{i} \epsilon_{i} U_{i}\right)=\nabla\left(\varepsilon_{i} \frac{\mu_{t}}{\sigma_{k}} \nabla \epsilon_{i}\right)+\frac{\varepsilon_{i}}{k}\left(C_{1 \epsilon} \varepsilon_{i} G_{k}-C_{2 \epsilon} \varepsilon_{i} \rho_{i} \epsilon_{i}\right)
$$

\subsubsection{Numerical Procedure}

The geometry of pretreatment reactor is shown in Figure 1. The numerical calculation used a two-dimensional mesh. The reactor had a diameter of $0.086 \mathrm{~m}$ and the height of $0.086 \mathrm{~m}$. To decrease the computational effort, and due to the reactor is symmetric, the reactor was split to become a half part. Initially, the acid-dilute pretreated samples settled at the reactor bottom to a level above the impeller, then sulfuric acid was injected to the rest of the reactor. The volume fraction of rice husk pretreated in the slurry entered to the reactor of $56 \%$, with 


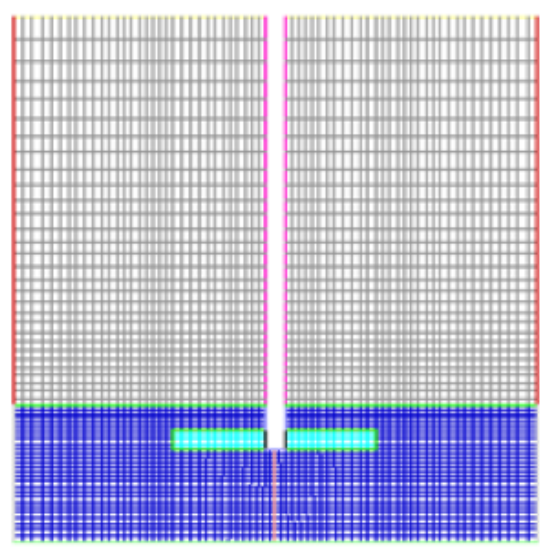

Figure 1. Two-dimensional geometry of pretreatment reactor

the average diameter of $60 \mu \mathrm{m}$. The operating conditions of reactor were the temperature of $85^{\circ} \mathrm{C}$ and the pressure of $1 \mathrm{~atm}$. A schematic of the reactor and the initial position of slurry solution are shown in Figure 1. Within the domain, there are three fluid zones, representing the impeller zone (green colours), the region where the slurry is initially located (blue colours) and the rest of tank where $\mathrm{NaOH}$ solution is filled (grey colours). The simulations were started using the initial assumptions similar to the specified volume fractions. The pretretreatment process was simulated for $60 \mathrm{~min}$. A constant time step of 0.01 was used. The simplification assumption is pretreated rice husk entered the reactor in the form of a slurry.

\section{RESULTS AND DISCUSSION}

\subsection{Morphological of the Rice Husk Structure}

Figure 2 shows the morphology of the rice husk structure (before pretreatment), b (after alkaline pretreatment), c (after alkaline-acid pretreatment). Major morphological changes of the rice husk (fig 2a) converted to fibrous clusters (fig $2 b$ ) were detected. It was shown by SEM that alterations in microstructure occurred. This is due to some lignin being eliminated by alkaline pretreatment. Lignin was also removed significantly during the alkaline-acid pretreatment (fig 2c). Thus the pretreatment of rice husk made it much more porous to enzymes. Cellulose and hemicellulose degradation occurred because of the reduction of lignin content and the alteration of its structural.

\subsection{Effect of the Combination of Alkaline and Acid Pre-} treatment on the rice husk composition

3.3 Effect of Single Pretreatment on the Lignin Content of Rice Husk

Lignin is the main problem for the utilization of second generation bioethanol. The lignocellulose wall lets as an inhibitor reducing the accessibility of cellulose to be hydrolysed. The lignin content must be reduced as much as possible to provide a high efficiency of pretreatment. As shown in Fig 3, Initially,

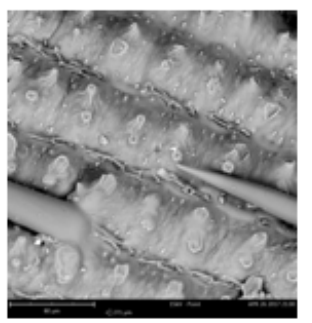

(a)

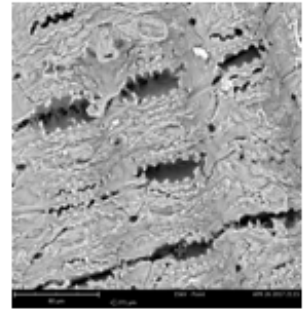

(b)

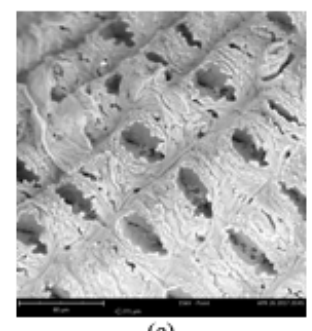

(c)

Figure 2. Morphology of the rice husk structure before pretreatment (a), after alkaline pretreatment (b), after alkaline-acid pretreatment (c)

the lignin content of rice husk was about $21.17 \%$. After acid pretreatment, the lowest lignin content was $10.74 \%$. Alkaline pretreatment produced the lowest lignin content of $4.35 \%$. Fig 3 also illustrated that the higher concentration of either $\mathrm{H}_{2} \mathrm{SO}_{4}$ or $\mathrm{NaOH}$ caused the increasing of lignin degradation. The highest capability of lignin degradation by acid pretreatment was about $38.38 \%$. Meanwhile, the alkaline pretreatment reduced the highest lignin content was about $55.34 \%$. It can be concluded that $\mathrm{H}_{2} \mathrm{SO}_{4}$ gave minor effect on lignin degradation level. On the other hand, $\mathrm{NaOH}$ lead the major effect on the degradation of lignin. Alkaline pretreatment broke down the lignin and glycoside bond on polysaccharide, so it can decrease the crystallinity. Previous researcher studied the lowest of lignin content is about $3.46 \%$ for solids pretreated at the acid concentration of $2 \%$ and 90 minutes of pretreatment time.

The lowest performance of alkaline pretreatment on a HWS (hot water solubility) of about $7.34 \%$ can be enhanced to become $9.71 \%$ by using combination alkaline-acid. HWS content was about $10.39 \%$. HWS content for both pretreatment was higher than acid pretreatment. This is due to $\mathrm{H}_{2} \mathrm{SO}_{4}$ formed emulsion at acid pretreatment. Meanwhile at the combination pretreatment, before $\mathrm{H}_{2} \mathrm{SO}_{4}$ form more emulsions, $\mathrm{NaOH}$ bonded with $\mathrm{H}_{2} \mathrm{SO}_{4}$ each other to become $\mathrm{NaSO}_{4}$ which is soluble in hot water. Fig 4 shows the both pretreatment combination degraded hemicellulose of about $9.59 \%$ (alkalineacid) and $9.27 \%$ (acid-alkaline). The highest cellulose content was about $66.75 \%$ for acid-alkaline pretreatment. For pretreatment combination, the lowest content of lignin was about $6.09 \%$ for acid-alkaline pretreatment.

It can be concluded that combination of either alkaline-acid or acid-alkaline had similar capability in increasing HWS, the 


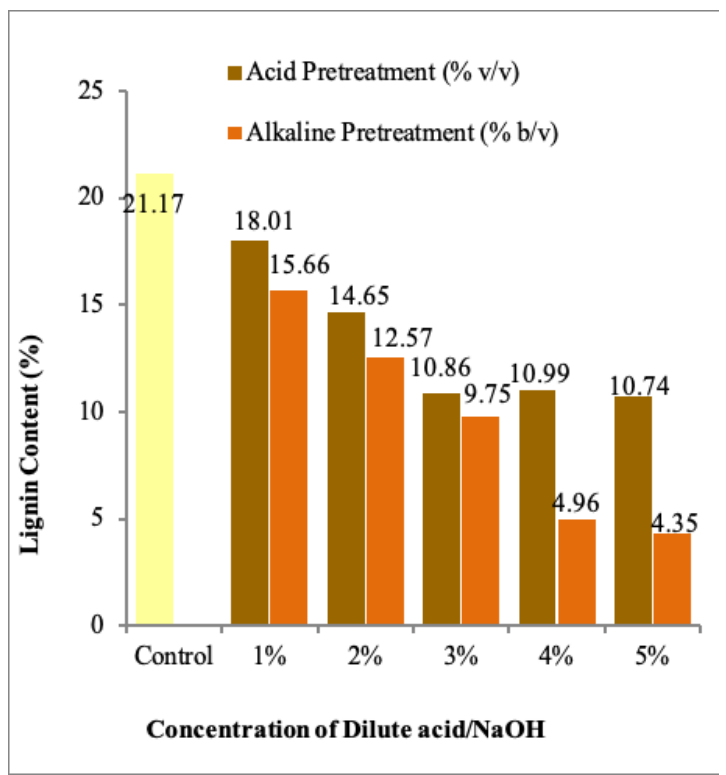

Figure 3. Effect of the Pretreatment on the Lignin Content of Rice Husk

degradation of hemicellulose, the enhancing of cellulose and the degradation of lignin. The difference of both pretreatment was about $\pm 1 \%$. Globally, acid-alkaline pretreatment was better than alkaline-acid pretreatment.

\subsection{Hydrodynamic Analysis of Pressure and Volume Frac- tion on Acid-Alkaline Pretreatment Using CFD (Ansys Fluent 16)}

To compare the simulation result and experimental data, this study focuses on hydrodunamics of acid-alkaline pretreatment. Figure 5 a shows the volume fraction of unpretreated rice husk. After pretreatment, the volume fraction of rice husk and reagent changed due to the mixing process. As illustrated in fig $5 \mathrm{c}$ and $5 \mathrm{~d}$, the impeller action caused the reagent in lower concentration (the blue color) and mixed with rice husk (red one). Furthermore, the rice husk at the lower level flowed to the upper one and mixed with reagent in the perfect mixing. The volume fraction of rice husk reduced because of mixing between rice husk and reagent solution. Figure $5 \mathrm{~b}$ describes the distribution of pressure after pretreatment. The blue color represents hydrostatic pressure normally; the highest pressure is represented by red color. Increasing of the pressure caused an enhancing of the higher kinetic energy turbulent which is desired in mixing of the rice husk and the reagent. We can see from the figure the small amount of blue color above the impeller. This area had a minimum pressure of about -628 $\mathrm{Pa}$ which is a vortex leading the minimum mixing efficiency. The highest pressure was about $8570 \mathrm{~Pa}$ (red color) where the maximal mixing occurred.

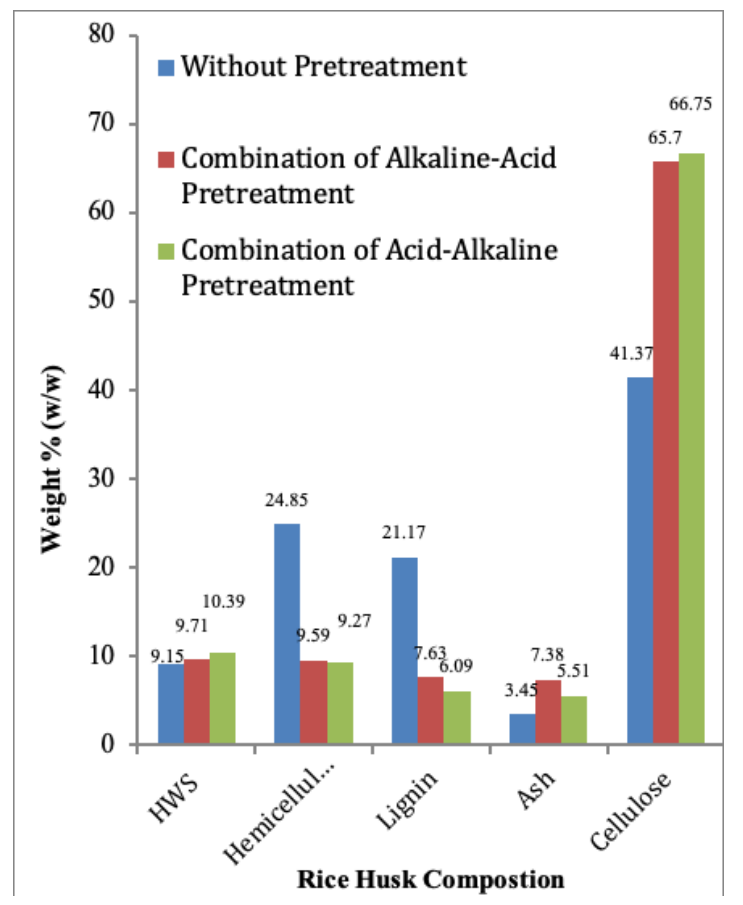

Figure 4. Effect of Different Pretreatment on the Composition of Rice Husk

\section{CONCLUSIONS}

A SEM-EDX investigation recorded that alterations in microstructure were observed, since a huge fraction of lignin was reduced by alkaline pretreatment. More lignin was also removed in the combined alkaline-acid pretreatment. After acid pretreatment, the lowest lignin content was $10.74 \%$. Alkaline pretreatment produced the lowest lignin content of $4.35 \%$. The highest capability of lignin degradation by acid pretreatment was about $38.38 \%$. Meanwhile, alkaline pretreatment reduced the highest lignin content to about $55.34 \%$.

The lower performance of alkaline pretreatment on HWS (hot water solubility) of about $7.34 \%$ can be enhanced to $9.71 \%$ using combined alkaline-acid. Both pretreatment combinations degraded hemicellulose of about $9.59 \%$ (alkaline-acid) and $9.27 \%$ (acid-alkaline). The highest cellulose content was about $66.75 \%$ for acid-alkaline pretreatment. The lowest content of lignin was about $6.09 \%$ for acid-alkaline pretreatment. It can be concluded that either the alkaline-acid or the acidalkaline combination had similar capability in increasing HWS, the degradation of hemicellulose, the enhancing of cellulose and the degradation of lignin. The mixing area had the minimum pressure of about $-628 \mathrm{~Pa}$ which is vortex leading the minimum mixing efficiency. The highest pressure was about $8570 \mathrm{~Pa}$ (red color) where the maximal mixing occurred. The rice husk flowed upward to the upper level and mixed with reagent in the perfect mixing. 

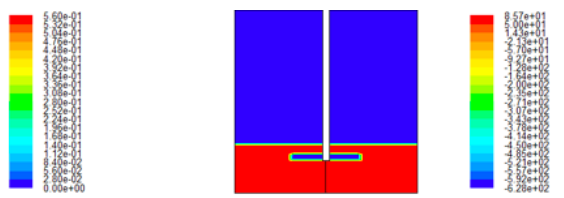

(a)
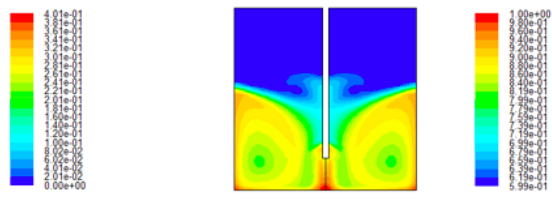

(c)

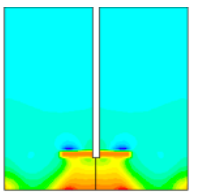

b)

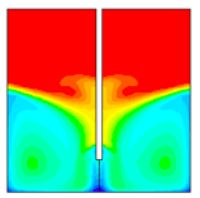

(d)
Figure 5. Initial Volume Fraction of Rice Husk (a); Contour Pressure (b); Volume Fraction of Rice Husk after Pretreatment (c); Volume Fraction of $\mathrm{NaOH}$ after Pretreatment (d)

\section{AGKNOWLEDGEMENT}

The authors would like to acknowledge supports from Universitas Sriwijaya for funding the research project (Unggulan Kompetitif Unsri, No. 1012/UN9.3.1/PP2017).

\section{REFERENCES}

Cara, C. e. a. (2006). Enhanced Enzymatic Hydrolysis of Olive Tree Wood by Steam Explosion and Alkaline Peroxide Delignification. Process Biochemistry, 41(2); 423-29

Chen, W.-H., B.-L. Pen, C.-T. Yu, and W.-S. Hwang (2011). Pretreatment efficiency and structural characterization of rice straw by an integrated process of dilute-acid and steam explosion for bioethanol production. Bioresource Technology, 102(3); 2916-2924

Cundari, L. e. a. (2016). Temperature Distribution of Biodiesel Blends Combustion in Boiler Using CFD-Fluent. International Journal on Advanced Science, Engineering and Information Technology, 6(1)

Fang, H., C. Zhao, and X.-Y. Song (2010). Optimization of enzymatic hydrolysis of steam-exploded corn stover by two approaches: Response surface methodology or using cellulase from mixed cultures of Trichoderma reesei RUTC30 and Aspergillus niger NL02. Bioresource Technology, 101(11); 4111-4119

Hamelinck, C. N., G. van Hooijdonk, and A. P. Faaij (2005). Ethanol from lignocellulosic biomass: techno-economic performance in short-, middle- and long-term. Biomass and Bioenergy, 28(4); 384-410

Harun, R., W. Jason, T. Cherrington, and M. K. Danquah (2011). Exploring alkaline pre-treatment of microalgal biomass for bioethanol production. Applied Energy, 88(10); 3464-3467

Hermansyah, Novia, M. Sugiyama, and S. Harashima (2015). Candida tropicalis Isolated from Tuak, a North SumateraIndonesian Traditional Beverage, for Bioethanol Production. Microbiology and Biotechnology Letters, 43(3); 241-248

Kaparaju, P. and C. Felby (2010). Characterization of lignin during oxidative and hydrothermal pre-treatment processes of wheat straw and corn stover. Bioresource Technology, 101(9); 3175-3181

Karimi, K., G. Emtiazi, and M. J. Taherzadeh (2006). Ethanol production from dilute-acid pretreated rice straw by simultaneous saccharification and fermentation with Mucor indicus, Rhizopus oryzae, and Saccharomyces cerevisiae. Enzyme and Microbial Technology, 40(1); 138-144

Kumar, S., U. Kothari, L. Kong, Y. Lee, and R. B. Gupta (2011). Hydrothermal pretreatment of switchgrass and corn stover for production of ethanol and carbon microspheres. Biomass and Bioenergy, 35(2); 956-968

Li, H., N.-J. Kim, M. Jiang, J. W. Kang, and H. N. Chang (2009). Simultaneous saccharification and fermentation of lignocellulosic residues pretreated with phosphoric acid-acetone for bioethanol production. Bioresource Technology, 100(13); 3245-3251

McIntosh, S. and T. Vancov (2010). Enhanced enzyme saccharification of Sorghum bicolor straw using dilute alkali pretreatment. Bioresource Technology, 101(17); 6718-6727

Mosier, N. (2005). Features of promising technologies for pretreatment of lignocellulosic biomass. Bioresource Technology, 96(6); 673-686

Novia, V. Pareek, and T. Agustina (2017). Bioethanol Production from Sodium Hydroxide - Dilute Sulfuric Acid Pretreatment of Rice Husk via Simultaneous Saccharification and Fermentation. In In MATEC Web of Conferences

Novia, N., S. Ray, , and V. Pareek (2007). Three-Dimensional Hydrodynamics and Reaction Kinetics Analysis in FCC Riser Reactors. Chemical Product and Process Modeling, 2(2); 4

Ohgren, Karin, R. Bura, J. Saddler, and G. Zacchi (2007). Effect of Hemicellulose and Lignin Removal on Enzymatic Hydrolysis of Steam Pretreated Corn Stover.". Bioresource Technology, 98(13); 2503-10

Sudiyani, Y., J. Waluyo, E. Triwahyuni, D. Burhani, Muryanto, P. Primandaru, A. P. Riandy, and N. Sumardi (2017). Optimization pretreatment condition of sweet sorghum bagasse for production of second generation bioethanol. In $A I P$ Conference Proceedings. Author(s)

Taherzadeh, M. J, and K. Karimi (2008). Pretreatment of Lignocellulosic Wastes to Improve Ethanol and Biogas Production: A Review. International Journal of Molecular Sciences 9(9):, 9(9); 1621-51

Thomsen, M. Hedegaard, A. Thygesen, and A. B. Thomsen (2008). Hydrothermal Treatment of Wheat Straw at Pilot Plant Scale Using a Three-Step Reactor System Aiming at High Hemicellulose Recovery, High Cellulose Digestibility and Low Lignin Hydrolysis. Bioresource Technology, 99(10); 4221-28

Xavier, A. M. R. B, M. F. Correia, S. R. Pereira, and D. V. Evtuguin (2010). Second-Generation Bioethanol from Eucalypt Sulphite Spent Liquor. Bioresource Technology, 101(8); 2755-61 